\title{
Finiteness Conditions for Fixed Point Iteration
}

\author{
Flemming Nielson, Hanne Riis Nielson \\ Computer Science Department, Aarhus University \\ Ny Munkegade, DK-8000 Aarhus C \\ Denmark \\ E-mail: \{fnielson,hrnielson\}@daimi.aau.dk
}

February 1992

\begin{abstract}
This paper provides a link between the formulation of static program analyses using the framework of abstract interpretation (popular for functional languages) and using the more classical framework of data flow analysis (popular for imperative languages). In particular we show how the classical notions of fastness, rapidity and $k$-boundedness carry over to the abstract interpretation framework and how this may be used to bound the number of times a functional should be unfolded in order to yield the fixed point. This is supplemented with a number of results on how to calculate the bounds for iterative forms (as for tail recursion), for linear forms (as for one nested recursive call), and for primitive recursive forms. In some cases this improves the "worst case" results of [9], but more importantly it gives much better "average case" results.
\end{abstract}

\section{Introduction}

In a recent paper [9] we gave precise bounds on a number $k$ such that 


$$
\text { FIX } H=H^{k} \perp
$$

where

$$
H:(A \rightarrow B) \rightarrow(A \rightarrow B)
$$

is a continuous functional corresponding to some static program analysis (or abstract interpretation). Here $A$ and $B$ are finite complete lattices and

$$
F I X=\lambda H . \bigsqcup\left\{H^{n} \perp \mid n \geq 0\right\}
$$

is the least fixed point operator. The development of [9] was sufficiently general to apply to imperative languages, where $A=B$ is a natural choice, as well as functional languages, where $A=\mathbf{2}^{p}$ and $B=\mathbf{2}$ is a natural choice for first-order strictness analysis.

In this paper we investigate functionals $H$ of the form

$$
\begin{aligned}
& H h=g_{0} \sqcup(G h) \\
& \text { where e.g. } G h=g \circ h \circ g_{1}
\end{aligned}
$$

and show that it is often possible to use special properties of $g_{0}, g$ and $g_{1}$ to obtain considerably lower bounds than the "worst-case" results of $[9]^{1}$. Even for the case of iterative forms, where $g=i d$, this may result in better bounds than those of [9, Section 4] provided that $g_{1}$ is sufficiently well-behaved.

However, one of the most interesting aspects of this work is that the kind of properties (of $g_{0}, g, g_{1}$ ) considered in this paper are close to the kind of properties studied in classical flow analysis [5]; this includes properties like fastness and $k$-boundedness. Thus at long last we seem to be able to bridge the gap between the fixed point techniques of the functions world and the imperative world! This was left open in [9] and [5, p.129], simply states: "determination of program properties by application of approximating semantics is often called abstract interpretation; this approach is formally (although not conceptually) equivalent to the algebraic framework approach presented [in [5]]".

\footnotetext{
${ }^{1}$ This is not intended to say that the "worst-case" bounds of [9] are wrong but rather that they may not arise for functionals $H$ of the form considered in this paper.
} 


\section{Overview of paper}

We begin by reviewing the lattice-theoretic notions that we will need (Section 2 ). Then we formulate the notions of fastness and $k$-boundedness [5] in the notation of abstract interpretation and we explore a few of their consequences (Section 3).

In Section 4 we then study "iterative forms" which are functionals $H$ of the form displayed above but with $g=i d$. We consider three cases, depending on whether the functions from $A$ to $B$ are

- total (written $A \rightarrow_{t} B$ )

- monotone (written $A \rightarrow_{m} B$ )

- strict and additive ${ }^{2}\left(\right.$ written $\left.A \rightarrow_{s a} B\right)$

In short we prove that the functional $G$ is $\ell_{\varphi}\left(g_{1}\right)$-bounded where $\varphi$ is one of $t, m$ or $s a$ and where $\ell_{\varphi}$ is some measure on functions. In practical terms this means that $F I X H=H^{k} \perp$ whenever $k \geq \ell_{\varphi}\left(g_{1}\right)$; thus only $\ell_{\varphi}\left(g_{1}\right)$ unfoldings of $H$ are needed.

In Section 5 we then study how to extend these results to "linear forms" which are functionals $H$ of the form displayed above but where $g$ is not restrained to be $i d$. A limitation of this development is that $g$ must be strict and additive for the results of Section 3 to be applicable. We can then show that $G$ is $\ell_{\varphi}(g) \cdot \ell_{\varphi}\left(g_{1}\right)$-bounded in the cases where $\varphi$ is $t$ or $m$. Unfortunately, the case where $\varphi$ is $s a$ eludes us.

The applicability of the results on "iterative forms" is further extended in Section 6 . Here we show that the program transformation technique of "accumulator introduction" may often be used to transform a functions in "primitive recursive form" into one in "iterative form". Fkthermore, if $k$ unfoldings suffice for the transformed functional then also $k$ unfoldings suffice for the original functional.

Finally, we compare our results with those of [9] and suggest directions for further research (Section 7). In the Appendix we characterize the "itera-

\footnotetext{
${ }^{2}$ In [5] additivity it is called "distributivity" and complete additivity (which is equvivalent to strictness and additivity given the finiteness of $A$ and $B$ ) is called "continuity".
} 
tive forms", "linear forms" and "primitive recursive forms" in terms of the functional programs and analyses for which they naturally arise.

An Extended Abstract appeared as [10].

\section{Preliminaries}

A finite complete lattice $(L, \sqsubseteq)$ is a finite set $L$, together with a partial order $\sqsubseteq$, such that each subset $Y$ of $L$ has a least upper bound; since a lest upper bound is unique (if it exists) it makes sense to write $\sqcup Y$ for it. It is customary to write $\perp=\sqcup \emptyset$ for the least element and $T=\sqcup L$ for the greatest element of $L$ and to write $l_{1} \sqcup l_{2}$ for $\sqcup\left\{l_{1}, l_{2}\right\}$. We shall write

$$
l_{1} \sqsubset l_{2} \text { for } l_{1} \sqsubseteq l_{2} \wedge l_{1} \neq l_{2}
$$

and we shall say that a chain

$$
l_{0} \sqsubset l_{1} \sqsubset \cdots \sqsubset l_{k}
$$

has length $k$ (rather than $k+1$ ). It is convenient to write

$\mathbf{C}(L)$ for the cardinality of $L$

$\mathbf{H}(L)$ for the height of $L$ (i.e. the length of the longest chain)

Writing $L \times L^{\prime}$ for the cartesian product and $L^{n}$ for the $n$-fold product $(n \geq 1)$ we have $\mathbf{C}\left(L \times L^{\prime}\right)=\mathbf{C}(L) \cdot \mathbf{C}\left(L^{\prime}\right), \mathbf{C}\left(L^{n}\right)=\mathbf{C}(L)^{n}, \mathbf{H}\left(L \times L^{\prime}\right)=\mathbf{H}(L)+$ $\mathbf{H}\left(L^{\prime}\right)$ and $\mathbf{H}\left(L^{n}\right)=n \cdot \mathbf{H}(L)$. Writing

$$
2=\int_{0}^{1}
$$


we have $\mathbf{C}(\mathbf{2})=2$ and $\mathbf{H}(\mathbf{2})=1$.

A function $f: L \rightarrow L^{\prime}$ from a finite complete lattice $L=(L, \sqsubseteq)$ to a finite complete lattice $L^{\prime}=\left(L^{\prime}, \sqsubseteq\right)$ is monotone if

$$
l_{1} \sqsubseteq l_{2} \Rightarrow f\left(l_{1}\right) \sqsubseteq f\left(l_{2}\right)
$$

and is strict if

$$
f(\perp)=\perp
$$

and is additive if

$$
f\left(l_{1} \sqcup l_{2}\right)=f\left(l_{1}\right) \sqcup f\left(l_{2}\right)
$$

Fact 1: A monotone function $f$ between finite complete lattices is continuous, i.e.

$$
f\left(\bigsqcup\left\{l_{n} \mid n \geq 0\right\}\right)=\bigsqcup\left\{f\left(l_{n}\right) \mid n \geq 0\right\}
$$

whenever $\forall n: l_{n} \sqsubseteq l_{n+1}$.

It is well known that

$$
F I X f=\bigsqcup\left\{f^{n}(\perp) \mid n \geq 0\right\}
$$

denotes the least fixed point of $f$ whenever $f$ is continuous; it thus follows that if $L$ is a finite complete lattice and $f: L \rightarrow L$ is monotone then $F I X f$ is the least fixed point of $f$.

Fact 2: A strict and additive function $f$ between finite complete lattices is completely additive, i.e.

$$
f(\bigsqcup Y)=\bigsqcup\{f(l) \mid l \in Y\}
$$

for all subsets $Y$.

We shall write $L \rightarrow_{t} L^{\prime}, L \rightarrow_{m} L^{\prime}$ and $L \rightarrow_{s a} L^{\prime}$ for the sets of total, monotone, and strict and additive functions from $L$ to $L^{\prime}$, respectively. The partial order is defined componentwise, i.e. 


$$
f \sqsubseteq f^{\prime} \text { if and only if } \forall l: f(l) \sqsubseteq f^{\prime}(l)
$$

and all of $L \rightarrow_{t} L^{\prime}, L \rightarrow_{m} L^{\prime}$ and $L \rightarrow_{s a} L^{\prime}$ will be finite complete lattices if $L$ and $L^{\prime}$ are. In all cases

$$
(\bigsqcup \mathcal{F})=\lambda l . \bigsqcup\{f(l) \mid f \in \mathcal{F}\}
$$

is the formula for least upper bounds.

Finally, a finite complete lattice $L$ is distributive [4] if

$$
l_{1} \sqcap\left(l_{2} \sqcup l_{3}\right)=\left(l_{1} \sqcap l_{2}\right) \sqcup\left(l_{1} \sqcap l_{3}\right)
$$

for all $l_{1}, l_{2}, l_{3} \in L$. An alternative characterization was given in $[9$, Lemma $17]$.

Fact 3: If $L$ and $L^{\prime}$ are distributive so are $L \times L^{\prime}, L^{n}, L \rightarrow_{t} L^{\prime}, L \rightarrow_{m} L^{\prime}$ and $L \rightarrow_{s a} L^{\prime}$.

Proof: Except for $L \rightarrow_{s a} L^{\prime}$ the results follow from the componentwise definition of $\sqcap$; also the results for $L \rightarrow_{t} L^{\prime}$ and $L \rightarrow_{m} L^{\prime}$ do not require the distributivity of $L$. In the case of $L \rightarrow_{s a} L^{\prime}$ we do not have a componentwise definition of $\sqcap$ and so instead we use the isomorphism between $L \rightarrow_{s a} L^{\prime}$ and $\mathcal{J}(L) \rightarrow_{s a} L^{\prime}$ where $\mathcal{J}(L)$ denotes the non-trivial join-irreducible elements of $L$; we refer to $[9$, Section 3.1] for (some of) the insights needed to prove this.

\section{Finiteness conditions on functionals}

Let $A$ and $B$ be arbitrary finite complete lattices and consider continuous (i.e. monotone) functionals

$$
G, H:\left(A \rightarrow_{\varphi} B\right) \rightarrow\left(A \rightarrow_{\varphi} B\right)
$$

where $\varphi$ is any one of $t, m$ or $s a$. Throughout this paper we shall assume that 


$$
H h=g_{0} \sqcup(G h)
$$

but in this section we shall not make any assumptions about the form of $G$. We are interested in determining the least fixed point, FIX $H$, of $H$ but before doing so we need a few definitions from classical flow analysis (as surveyed in [5]).

As usual $G^{i}(h)$ denotes the $i$-fold iteration of $G$ so that $G^{0}(h)=h$ and $G^{i+1}(h)=G\left(G^{i}(h)\right)=G^{i}(G(h))$. It is convenient to write Id for the functional defined by $\operatorname{Id}(h)=h$. We then define

$$
G^{i}(h)=\bigsqcup\left\{G^{j}(h) \mid 0 \leq j<i\right\}
$$

This is well-defined given the assumptions on $A$ and $B$ and the results of Section 2. Concerning $G^{[i+1]}$ one may calculate that

$$
G^{[i+1]}(h)=h \sqcup \cdots \sqcup G^{i}(h)
$$

so that $G^{[i+1]}=I d \sqcup\left(G^{[i]} \circ G\right)$. We also have

Fact 4: If $G$ is strict and additive then $G^{[i+1]}=I d \sqcup\left(G \circ G^{[i]}\right)$.

Fact 5: If $G \sqsupseteq I d$ then $G^{[i+1]}=G^{i} \sqsupseteq I d$; it follows that we also have $G^{[i+2]}=I d \sqcup\left(G \circ G^{[i+1]}\right)$.

Clearly $G \sqsubseteq G \sqcup I d$ so that also $G^{[i]} \sqsubseteq(G \sqcup I d)^{[i]}$ for all $i$. For equality we note

Fact 6: $G^{[i]}=(G \sqcup I d)^{[i]}$ if $G$ is strict and additive (or if $G \sqsupseteq I d$ ).

Proof: If $G \sqsupseteq I d$ we have $G=G \sqcup I d$ and the result is immediate.

If $G$ is strict and additive we have $G^{[0]}=\perp=(G \sqcup I d)^{[0]}$ and

$$
G^{[i+1]}=\bigsqcup\left\{G^{j} \mid 0 \leq j \leq i\right\}=(G \sqcup I d)^{i}=(G \sqcup I d)^{[i+1]}
$$

and this concludes the proof.

The interest in $G^{[i]}$ can now be motivated by:

Lemma 7: FIX $H=\bigsqcup\left\{(G \sqcup I d)^{[i]}\left(g_{0}\right) \mid i \geq 0\right\}$

Proof: It is helpful to write $G^{\{i\}}=(G \sqcup I d)^{[i]}$. We first prove 
- $H^{i}(\perp) \sqsubseteq G^{\{i+1\}}\left(g_{0}\right)$

by numerical induction on $i$. The base case, $i=0$, is immediate and for the inductive steps $i=j+1$, we calculate

$$
\begin{aligned}
H^{j+1}(\perp) & =g_{0} \sqcup G\left(H^{j} \perp\right) \\
& \sqsubseteq g_{0} \sqcup G\left(G^{\{j+1\}}\left(g_{0}\right)\right) \\
& \sqsubseteq G^{\{j+1\}}\left(g_{0}\right) \sqcup G\left(G^{\{j+1\}}\left(g_{0}\right)\right) \\
& =(G \sqcup I d)\left(G^{\{j+1\}}\left(g_{0}\right)\right) \\
& =G^{\{j+2\}}\left(g_{0}\right)
\end{aligned}
$$

where we have used the monotonicity of $G$, the induction hypothesis and Fact 5 (with $G \sqcup I d \sqsupseteq I d$ ).

We next prove

- $G^{\{i\}}\left(g_{0}\right) \sqsubseteq F I X \quad H$

It is convenient to take $i=0$ and $i=1$ as base cases and in both cases the result is immediate because $G^{\{0\}}\left(g_{0}\right)=\perp$ and $G^{\{1\}}\left(g_{0}\right)=g_{0}$. For the inductive step, $i=j+2$, we calculate

$$
\begin{aligned}
& G^{\{j+2\}}\left(g_{0}\right) \quad=(G \sqcup I d)\left(G^{\{j+1\}}\left(g_{0}\right)\right) \\
& \sqsubseteq(G \sqcup I d)(F I X \quad H) \\
& \sqsubseteq(\text { FIX } H) \sqcup g_{0} \sqcup G(\text { FIX } H) \\
& =(\text { FIX } H) \sqcup H(F I X H) \\
& =\text { FIX } H
\end{aligned}
$$

This ends the proof of the Lemma.

Corollary 8: If $G$ is strict and additive we have

$$
F I X H=\bigsqcup\left\{G^{[i]}\left(g_{0}\right) \mid i \geq 0\right\}
$$

(This also holds if $G \sqsupseteq I d$.)

Following [5] we shall say that $G$ is $k$-bounded if 


$$
\forall h \in A \rightarrow_{\varphi} B: G^{[k+1]}(h)=G^{[k]}(h)
$$

There is a related concept called $k$-semi-boundedness but for strict (and additive) functionals it is equivalent to $k$-boundedness and so will not be of interest in this paper. The special case of 2-boundedness is known as fastness; in [5] this is motivated by the observation that for a fast problem only one iteration around the loop body will be needed.

Fact 9: If $G$ is $k$-bounded then $G^{[i]}(h)=G^{[k]}(h)$ for all $i \geq k$ and for all $h \in A \rightarrow_{\varphi} B$.

Proof: Use induction on $i \geq k$.

Turning to the consequences for the least fixed point, FIX $H$, of $H$ we then have:

Fact 10: If $G$ is strict and additive then $H^{i} \perp=G^{[i]}\left(g_{0}\right)$.

Proof: The equality may be shown by numerical induction on $i$. Only the inductive step is non-trivial and here we calculate

$$
\begin{aligned}
H^{i+1} \perp & =g_{0} \sqcup G\left(H^{i} \perp\right) \\
& =g_{0} \sqcup G\left(G^{[i]}\left(g_{0}\right)\right) \\
& =\left(I d \sqcup\left(G \circ G^{[i]}\right)\right)\left(g_{0}\right) \\
& =G^{[i+1]}\left(g_{0}\right)
\end{aligned}
$$

where the last step is using Fact 4.

Lemma 11: If $G$ is strict and additive and $G$ is $k$-bounded then

$$
F I X H=G^{[k]}\left(g_{0}\right)=H^{k}(\perp)
$$

The $k$-boundedness of $G$ may be weakened to $G^{[k+1]}\left(g_{0}\right)=G^{[k]}\left(g_{0}\right)$.

For the applicability of the results of the present paper, in particular Lemma 11 , it is important that the functional $G$ is

- $k$-bounded, and

- strict and additive 
We will develop formulae for determining the constant $k$ in Sections 4, 5 and 6.

Here we will conclude with a few examples of how to manufacture strict and additive functionals. Consider the following definitions of functionals $G:\left(A \rightarrow_{\varphi} B\right) \rightarrow\left(A \rightarrow_{\varphi} B\right):$

- $G h=h \circ g_{1}$

- $G h=g \circ h \quad$ where $g$ is strict and additive

- $G=G_{1} \circ G_{2} \quad$ where $G_{1}$ and $G_{2}$ are strict and additive

- $G=G_{1} \sqcup G_{2} \quad$ where $G_{1}$ and $G_{2}$ are strict and additive

- $G h=g \sqcap h \quad$ where $A \rightarrow_{\varphi} B$ is distributive

- $G h=\operatorname{tuple}\left(G_{1} h, G_{2} h\right)=\lambda l$. $<G_{1} h l, G_{2} h l>$ where $G_{1}$ and $G_{2}$ are strict and additive

- $G h=g \circ h \circ g_{1} \quad$ where $g$ is strict and additive

- $G h=g \circ$ tuple $\left(h \circ g_{1}, g_{2}\right)$

where $g$ is strict and additive in its left

argument, i.e. $g(\perp, l)=\perp$ and

$g\left(l_{1} \sqcup l_{2}, l\right)=g\left(l_{1}, l\right) \sqcup g\left(l_{2}, l\right)$

Then $G$ is strict and additive in all cases. We shall use the first and the two last observations in Sections 4, 5 and 6 respectively.

\section{Iterative forms}

We now study a functional $G:\left(A \rightarrow_{\varphi} B\right) \rightarrow\left(A \rightarrow_{\varphi} B\right)$ defined by

$$
G h=h \circ g_{1}
$$

for $g_{1} \in A \rightarrow_{\varphi} B$. Clearly $G$ is continuous (i.e. monotone), strict and additive; this means that for $H$ defined by $H h=g_{0} \sqcup(G h)$ we will have FIX $H=G^{[k]}\left(g_{0}\right)=H^{k}(\perp)$ whenever $G$ is $k$-bounded. In this section we shall define three measures on functions (denoted $\ell_{t}, \ell_{m}$ and $\ell_{s a}$ ) and we shall show that $G$ will be $\ell_{\varphi}\left(g_{1}\right)$-bounded. This will be illustrated on an accumulator version of the factorial program.

The first case is where $\varphi$ is $t$. Here we define 


$$
\begin{aligned}
& \ell_{t}(f, x)=\min \left\{k \mid f^{k}(x) \in\left\{x, \ldots, f f^{k-1}(x)\right\}, k>0\right\} \\
& \ell_{t}(f)=\max \left\{\ell_{t}(f, x) \mid x \in A\right\}
\end{aligned}
$$

and note

Fact 12: $1 \leq \ell_{t}(f, x) \leq \ell_{t}(f) \leq \mathrm{C}(A)$ when $f: A \rightarrow_{t} A$ and $x \in A$.

We then have

Lemma 13: The functional $G:\left(A \rightarrow_{t} B\right) \rightarrow\left(A \rightarrow_{t} B\right)$ defined by $G h=$ $h \circ g_{1}$ is $\ell_{t}\left(g_{1}\right)$-bounded.

Proof: Setting $k=\ell_{t}\left(g_{1}\right)$ we must prove that $G^{[k+1]}=G^{[k]}$ and for this it suffices to prove $G^{k} \sqsubseteq G^{[k]}$. So let $h \in A \rightarrow_{t} B$ and $w \in A$ be given and note that

$$
G^{k} h w=h\left(g_{1}^{k}(w)\right)
$$

and that

$$
G^{[k]} h w=\bigsqcup\left\{h(w), \ldots, h\left(g_{1}^{k-1}(w)\right)\right\}
$$

(where we used $k>0$ ). Fkom the definition of $k$ there exists $i$ such that $0 \leq i<k$ and $g_{1}^{k}(w)=g_{1}^{i}(w)$. Hence

$$
G^{k} h w=h\left(g_{1}^{i}(w)\right)
$$

where $i \leq k-1$ and the result is immediate.

Example 14: An accumulator version of the factorial program may be written as follows

$$
\operatorname{fac}(n, a)=\text { if } n=0 \text { then } a \text { else } \operatorname{fac}(n-1, n * a)
$$

where the initial call is $\operatorname{fac}(n, 1)$. We shall not go into the details of how to perform a strictness analysis for this program but simply postulate (or see the Appendix) that it may be obtained as follows: 


$$
\begin{aligned}
& A=\mathbf{2} \times \mathbf{2} \\
& B=\mathbf{2} \\
& g_{0}\left(n^{\#}, a^{\#}\right)=n^{\#} \sqcap a^{\#} \\
& g_{1}\left(n^{\#}, a^{\#}\right)=\left(n^{\#}, n^{\#} \sqcap a^{\#}\right)
\end{aligned}
$$

where $n^{\#} \sqcap a^{\#}$ denotes the greatest lower bound of $\left\{n^{\#}, a^{\#}\right\}$, i.e.

$$
n^{\#} \sqcap a^{\#}= \begin{cases}1 & \text { if } n^{\#}=1=a^{\#} \\ 0 & \text { otherwise }\end{cases}
$$

Thus $G h=h \circ g_{1}$ and $H h=g_{0} \sqcup(G h)$ and we must determine $k$ such that FIX $H=G^{[k]}\left(g_{0}\right)=H^{k} \perp$.

The results of $[9$, Section 3] are applicable and ensure that one may take

$$
k=\mathbf{H}\left(A \rightarrow_{m} B\right)=\mathbf{C}(A) \cdot \mathbf{H}(B)=4 \cdot 1=4
$$

Using Lemmas 13 and 11 we may improve this by taking

$$
k=\ell_{t}\left(g_{1}\right)=2
$$

That $\ell_{t}\left(g_{1}\right)=2$ follows easily from the idempotence of $g_{1}$, i.e. $g_{1} \circ g_{1}=g_{1}$.

This result is not optimal, however. Tabulating $H^{i} \perp w$ for $i \in\{0,1,2\}$ and $w \in A=\{(0,0),(0,1),(1,0),(1,1)\}$ one gets

\begin{tabular}{|l||c|c|c|c|}
\hline$w$ & $(0,0)$ & $(0,1)$ & $(1,0)$ & $(1,1)$ \\
\hline \hline$H^{0} \perp w$ & 0 & 0 & 0 & 0 \\
$H^{1} \perp w$ & 0 & 0 & 0 & 1 \\
$H^{2} \perp w$ & 0 & 0 & 0 & 1 \\
\hline
\end{tabular}

and this shows that it is possible to use $k=1$ in this example.

The second case is where $\varphi$ is $m$. Here we first need to define the set $L C(Y)$ of elements less than (or equal to) some element of $Y$, i.e.

$$
L C(Y)=\left\{x \in A \mid \exists x^{\prime} \in Y: x \sqsubseteq x^{\prime}\right\}
$$


We then define

$$
\begin{aligned}
& \ell_{m}(f, x)=\min \left\{k \mid f^{k}(x) \in L C\left(\left\{x, \ldots, f^{k-1}(x)\right\}\right), k>0\right\} \\
& \ell_{m}(f)=\max \left\{\ell_{m}(f, x) \mid x \in A\right\}
\end{aligned}
$$

Fact 15: $1 \leq \ell_{m}(f, x) \leq \ell_{m}(f) \leq \mathbf{C}(A)$ and $\ell_{m}(f) \leq \ell_{t}(f)$ whenever $f: A \rightarrow_{m} A$ and $x \in A$.

We then have

Lemma 16: The functional $G:\left(A \rightarrow_{m} B\right) \rightarrow\left(A \rightarrow_{m} B\right)$ defined by $G h=h \circ g_{1}$ is $\ell_{m}\left(g_{1}\right)$-bounded.

Proof: Setting $k=\ell_{m}\left(g_{1}\right)$ we must prove that $G^{[k+1]}=G^{[k]}$ and for this it is sufficient to prove $G^{k} \sqsubseteq G^{[k]}$. So let $h \in A \rightarrow_{m} B$ and $w \in A$ be given and note that

$$
G^{k} h w=h\left(g_{1}^{k}(w)\right)
$$

and that

$$
G^{[k]} h w=\bigsqcup\left\{h(w), \ldots, h\left(g_{1}^{k-1}(w)\right)\right\}
$$

(where we used $k>0$ ). From the definition of $k$ there exists $i$ such that $0 \leq i<k$ and $g_{i}^{k}(w) \sqsubseteq g_{1}^{i}(w)$. Hence

$$
G^{k} h w \sqsubseteq h\left(g_{1}^{i}(w)\right)
$$

where $i \leq k-1$ and the result is immediate.

Example 17: Continuing the previous example we now may take

$$
k=\ell_{m}\left(g_{1}\right)=1
$$

which is the optimal result. To see that $\ell_{m}\left(g_{1}\right)=1$ simply note that $g_{1}$ is reductive, i.e. $g_{1} \sqsubseteq i d$.

The third case is where $\varphi$ is $s a$. Here we define 


$$
\begin{aligned}
& \ell_{s a}(f, x)=\min \left\{k \mid f^{k}(x) \sqsubseteq \bigsqcup\left\{x, \ldots, f^{k-1}(x)\right\} k>0\right\} \\
& \ell_{s a}(f)=\max \left\{\ell_{s a}|(f, x)| x, \in A\right\}
\end{aligned}
$$

and note

Fact 18: $1 \leq \ell_{s a}(f, x) \leq \ell_{s a}(f) \leq \mathbf{H}(A)$ and $\ell_{s a}(f) \leq \ell_{m}(f)$ whenever $f \in A \rightarrow_{s a} A$ and $x \in A$; here we assume that $\mathbf{H}(A)>0$ is indeed the case.

Proof: The only non-trivial proof is to show that $\ell_{s a}(f, x) \leq \mathbf{H}(A)$ rather than e.g. $\ell_{s a}(f, x) \leq \mathbf{H}(A)+1$. For this note that

$$
\ell_{s a}(f, \perp)=1
$$

and that if $x \neq \perp$ then

$$
\perp \sqsubset x \sqsubset(x \sqcup f(x)) \sqsubset \cdots \sqsubset\left(x \sqcup f(x) \sqcup \cdots \sqcup f^{k-1}(x)\right)
$$

is a proper chain when $k=\ell_{s a}(f, x)$. Hence $k \leq \mathbf{H}(A)$ and this establishes the result.

We then have

Lemma 19: The functional $G:\left(A \rightarrow_{s a} B\right) \rightarrow\left(A \rightarrow_{s a} B\right)$ defined by $G h=h \circ g_{1}$ is $\ell_{s a}\left(g_{1}\right)$-bounded.

Proof: Setting $k=\ell_{s a}\left(g_{1}\right)$ we must prove that $G^{[k+1]}=G^{[k]}$ and for this it is sufficient to prove $G^{k} \sqsubseteq G^{[k]}$. So let $h \in A \rightarrow_{s a} B$ and $w \in A$ be - given and note that

$$
G^{k} h w=h\left(g_{1}^{k}(w)\right)
$$

and that

$$
\begin{aligned}
G^{[k]} h w & =\bigsqcup\left\{h(w), \ldots, h\left(g_{1}^{k-1}(w)\right)\right\} \\
& =h\left(\bigsqcup\left\{w, \ldots g_{1}^{k-1}(w)\right\}\right)
\end{aligned}
$$

where we have used that $k>0$ and that $h$ is completely additive. From the definition of $k$ we have 


$$
g_{1}^{k}(w) \sqsubseteq \bigsqcup\left\{w, \ldots, g_{1}^{k-1}(w)\right\}
$$

and (by monotonicity of $h$ ) the result follows.

From Fact 18 and Lemmas 19 and 11 it follows that

$$
F I X H=H^{k} \perp \text { for } k=\mathbf{H}(A)
$$

in the strict and additive case. It is interesting to note that this is the (tight) upper bound established in [9, Section 4] for functionals in iterative form.

Example 20: Continuing the previous example we note that

$$
\ell_{s a}\left(g_{1}\right)=1=\ell_{m}\left(g_{1}\right)
$$

however, $g_{1}$ is not additive so the above lemma is not applicable.

Example 21: In certain cases the bound obtained from Lemma 19 will indeed be better than that obtained from Lemma 16. To illustrate this we shall consider a detection of signs analysis which is a typical example of an analysis in the strict and additive framework. The analysis will be based on the lattice $\mathbf{S}$ of signs depicted below:

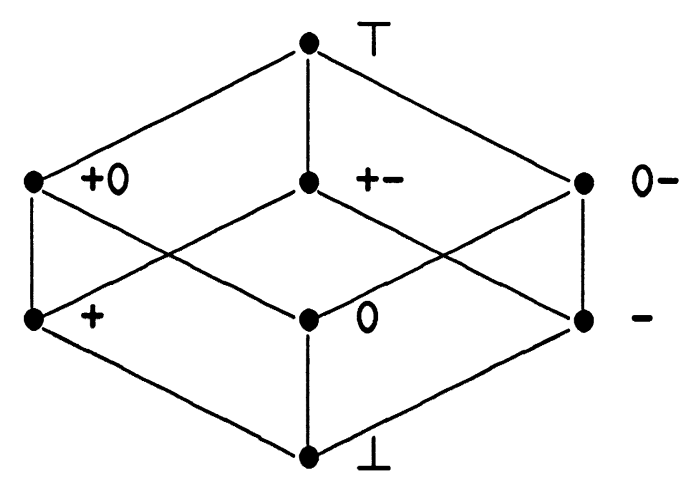

Here + describes the positive natural numbers, 0 the natural number $0,+0$ the non-negative natural numbers etc.

Now consider the function 


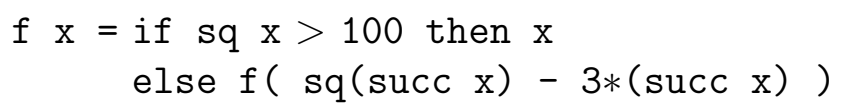

The analysis of $\mathrm{f}$ will give rise to a functional

$$
H:(\mathbf{S} \rightarrow \mathbf{S}) \rightarrow(\mathbf{S} \rightarrow \mathbf{S})
$$

in iterative form, i.e.

$$
H h=g_{0} \sqcup h \circ g_{1}
$$

Using the "obvious" interpretation of the primitives sq, >, succ, - and $*$ we may obtain the following definitions of $g_{0}$ and $g_{1}$ :

\begin{tabular}{|l||cccccccc|}
\hline$x$ & $\perp$ & + & 0 & - & +0 & +- & $0-$ & $\top$ \\
\hline \hline$g_{0}(x)$ & $\perp$ & + & $\perp$ & - & +0 & +- & $0-$ & $\top$ \\
$g_{1}(x)$ & $\perp$ & $\top$ & $\top$ & $0+$ & $\top$ & $\top$ & $\top$ & $\top$ \\
\hline
\end{tabular}

We have

$$
\ell_{t}\left(g_{1}\right)=1=\ell_{m}\left(g_{1}\right)=3
$$

so Lemma 16 gives that 3 iterations will suffice for computing the fixed point. However

$$
\ell_{s a}\left(g_{1}\right)=2
$$

so Lemma 19 shows than an even better bound can be obtained.

Sometimes simple "algebraic" properties of functions, like idempotency, may be used to obtain bounds on $\ell_{\varphi}$. The following table summarizes some such results; whenever

- $f \in A \rightarrow_{\varphi} A$ 
- $f$ satisfies the condition in the left column

the corresponding table entry gives

- an upper bound on $\ell_{\varphi}(f)$

\begin{tabular}{|l||ccc|}
\hline & $\varphi=t$ & $\varphi=m$ & $\varphi=s a$ \\
\hline \hline$f=f$ (worst-case) & $\mathbf{C}(A)$ & $\mathbf{C}(A)$ & $\mathbf{H}(A)$ \\
$f=i d$ (trivial case) & 1 & 1 & 1 \\
$f=f \circ f$ (idempotence) & 2 & 2 & 2 \\
$f \sqsupseteq i d$ (extensive) & $\mathbf{H}(A)+1$ & $\mathbf{H}(A)+1$ & $\mathbf{H}(A)$ \\
$f \sqsubseteq i d$ (reductive) & $\mathbf{H}(A)+1$ & 1 & 1 \\
$f \circ f \sqsubseteq f$ (weak idempotence) & $\mathbf{C}(A)$ & 2 & 2 \\
$f \circ f \sqsubseteq f \sqcup i d$ (fast) & $\mathbf{C}(A)$ & $\mathbf{C}(A)$ & 2 \\
\hline \hline$f^{k}=f^{i}$ for $i<k$ & $k$ & $k$ & $k$ \\
$f^{k} \sqsubseteq f^{i}$ for $i<k$ & $\mathbf{C}(A)$ & $k$ & $k$ \\
$f^{k} \sqsubseteq f^{[k]}$ & $\mathbf{C}(A)$ & $\mathbf{C}(A)$ & $k$ \\
\hline
\end{tabular}

\section{Linear forms}

We now increase our level of ambition and study a functions

$$
G:\left(A \rightarrow_{\varphi} B\right) \rightarrow\left(A \rightarrow_{\varphi} B\right)
$$

defined by

$$
G h=g \circ h \circ g_{1}
$$

for $g \in B \rightarrow_{\varphi} B$ and $g_{1} \in A \rightarrow_{\varphi} A$. This functional is continuous (i.e. monotone) if $g$ is monotone.

For the results of Section 3 to be applicable we need $G$ not only to be continuous but also strict and additive and this holds when $g$ is strict and additive; then the function $H$ defined by $H \quad h=g_{0} \sqcup(G h)$ will have FIX $H=G^{[k]}\left(g_{0}\right)=H^{k}(\perp)$ whenever $G$ is $k$-bounded. However, in this 
section we shall not formally assume that $g$ is strict and additive as the results on $k$-boundedness of $G$ do not depend on this.

The first case is where $\varphi$ is $t$. Using the measure $\ell_{t}$ of the previous section we have

Lemma 22: The functional $G:\left(A \rightarrow_{t} B\right) \rightarrow\left(A \rightarrow_{t} B\right)$ defined by $G h=$ $g \circ h \circ g_{1}$ is $\ell_{t}(g) \cdot \ell_{t}\left(g_{1}\right)$-bounded.

Before approaching the proof we need an auxiliary notion. For a function $f \in A \rightarrow_{t} B$ and an element $x \in A$ define

$$
\varrho_{t}(f, x)=\max \left\{i \mid f^{k}(x) \in\left\{x, f(x), \ldots, f^{k-i}(x)\right\}, k=\ell_{t}(f, x)\right\}
$$

and note that

$$
1 \leq \varrho_{t}(f, x) \leq \ell_{t}(f, x)
$$

Fact 23: If $k \geq \ell_{t}(f, x)$ then $f^{k}(x)=f^{k-\varrho_{t}(f, x)}(x)$.

Proof: We proceed by induction on $k \geq \ell_{t}(f, x)$. The case $k=\ell_{t}(f, x)$ follows from the definition of $\varrho_{t}(f, x)$. For the induction step we write $\varrho=$ $\varrho_{t}(f, x)$ and calculate

$$
f^{k+1}(x)=f\left(f^{k}(x)\right)=f\left(f^{k-\varrho}(x)\right)=f^{(k+1)-\varrho}(x)
$$

This completes the proof.

Fact 24: If $k-n \cdot \varrho_{t}(f, x) \geq \ell_{t}(f, x)-\varrho_{t}(f, x)$ and $n \geq 0$ then $f^{k}(x)=$ $f^{k-n \cdot \varrho_{t}(f, x)}(x)$.

Proof: We write $\varrho=\varrho_{t}(f, x)$ and proceed by induction on $n$. The case $n=0$ is trivial and for the induction step we assume that $k-(n+1) \cdot \varrho \geq \ell_{t}(f, x)-\varrho$. Then $k-n \cdot \varrho \geq \ell_{t}(f, x) \geq \ell_{t}(f, x)-\varrho$ and the induction hypothesis gives the first equality of

$$
f^{k}(x)=f^{k-n \cdot \varrho}(x)=f^{k-n \cdot \varrho-\varrho}(x)
$$

whereas the second equality follows from Fact 23. This completes the proof. 
Proof of Lemma 22: Setting $k=\ell_{t}(g) \cdot \ell_{t}\left(g_{1}\right)$ we must prove $G^{[k+1]}=G^{[k]}$ and for this it suffices to prove $G^{k} \sqsubseteq G^{[k]}$. So assume that $h \in A \rightarrow_{t} B$ and $w \in A$ are given and show that

$$
G^{k} h w \sqsubseteq G^{[k]} h w
$$

It suffices to find $k^{\prime}$ such that $0 \leq k-k^{\prime}<k$ (i.e. $1 \leq k^{\prime} \leq k$ ) and

$$
G^{k} h w \sqsubseteq G^{k-k^{\prime}} h w
$$

Now let

$$
k^{\prime}=\varrho_{t}\left(g, h\left(g_{1}^{k} w\right)\right) \cdot \varrho_{t}\left(g_{1}, w\right)
$$

We then have

$$
\begin{aligned}
k-k^{\prime} & =\ell_{t}(g) \cdot \ell_{t}\left(g_{1}\right)-\varrho_{t}\left(g, h\left(g_{1}^{k}(w)\right)\right) \cdot \varrho_{t}\left(g_{1}, w\right) \\
& \geq \varrho_{t}\left(g, h\left(g_{1}^{k}(w)\right)\right) \cdot \ell_{t}\left(g_{1}, w\right)-\varrho_{t}\left(g, h\left(g_{1}^{k}(w)\right)\right) \cdot \varrho_{t}\left(g_{1}, w\right) \\
& =\varrho_{t}\left(g, h\left(g_{1}^{k}(w)\right)\right) \cdot\left(\ell_{t}\left(g_{1}, w\right)-\varrho_{t}\left(g_{1}, w\right)\right) \\
& \geq \ell_{t}\left(g_{1}, w\right)-\varrho_{t}\left(g_{1}, w\right)
\end{aligned}
$$

and similarly

$$
\left.k-k^{\prime} \geq \ell_{t}\left(g, h\left(g_{1}^{k}(w)\right)\right)-\varrho_{t}\left(g, h\left(g_{1}^{k}, w\right)\right)\right)
$$

Using Fact 24 we then get

$$
\begin{aligned}
G^{k} h w & =g^{k}\left(h\left(g_{1}^{k}(w)\right)\right) \\
& =g^{k-k^{\prime}}\left(h\left(g_{1}^{k}(w)\right)\right) \\
& =g^{k-k^{\prime}}\left(h\left(g_{1}^{k-k^{\prime}}(w)\right)\right) \\
& =G^{k-k^{\prime}} h w
\end{aligned}
$$

This proves the lemma. 
The second case is where $\varphi$ is $m$. Using the measure $\ell_{m}$ of the previous section we get

Lemma 25: The functional $G:\left(A \rightarrow_{m} B\right) \rightarrow\left(A \rightarrow_{m} B\right)$ defined by $G h=g \circ h \circ g_{1}$ (for $g \in B \rightarrow_{m} B$ and $g_{1} \in A \rightarrow_{m} A$ ) is $\ell_{m}(g) \cdot \ell_{m}\left(g_{1^{-}}\right.$ bounded.

Again we shall need som auxiliary results before giving the proof. Define

$$
\begin{aligned}
& \varrho_{m}(f, x)=\max \left\{i \mid f^{k}(x) \in L C\left(\left\{x, f(x), \ldots, f^{k-i}(x)\right\}\right), k=\right. \\
& \left.\ell_{m}(f, x)\right\}
\end{aligned}
$$

and observe that

$$
1 \leq \varrho_{m}(f, x) \leq \ell_{m}(f, x)
$$

Fact 26: If $k \geq \ell_{m}(f, x)$ then $f^{k}(x) \sqsubseteq f^{k-\varrho_{m}(f, x)}(x)$.

Proof: We proceed by induction on $k \geq \ell_{m}(f, x)$. The case $k=\ell_{m}(f, x)$ follows from the definition of $\varrho_{m}(f, x)$. For the induction step we write $\varrho=$ $\varrho_{m}(f, x)$ and calculate

$$
f^{k+1}(x)=f\left(f^{k}(x)\right) \sqsubseteq f\left(f^{k-\varrho}(x)\right)=f^{(k+1)-\varrho}(x)
$$

where we have used the monotonicity of $f$.

Fact 27: If $k-n \cdot \varrho_{m}(f, x) \geq \ell_{m}(f, x)-\varrho_{m}(f, x)$ and $n \geq 0$ then $f^{k}(x) \sqsubseteq$ $f^{k-n \cdot \varrho_{m}(f, x)}(x)$.

Proof: We write $\varrho=\varrho_{m}(f, x)$ and proceed by induction on $n$. The case $n=0$ is trivial and for the induction step we assume that $k-(n+1) \cdot \varrho \geq \ell_{m}(f, x)-\varrho$. Then $k-n \cdot \varrho \geq \ell_{m}(f, x) \geq \ell_{m}(f, x)-\varrho$ and using the induction hypothesis and Fact 26 we get

$$
f^{k}(x)=f^{k-n \cdot \varrho}(x) \sqsubseteq f^{k-n \cdot \varrho-\varrho}(x)
$$

as required.

Proof of Lemma 25: As in the proof of Lemma 22 we may let $h \in A \rightarrow_{m} B$ and $w \in A$ be given and then show that 


$$
G^{k} h w \sqsubseteq G^{[k]} h w
$$

for $k=\ell_{m}(g) \cdot \ell_{m}\left(g_{1}\right)$.

For this it suffices to find $k^{\prime}$ such that $0 \leq k-k^{\prime}<k$ (i.e. $1 \leq k^{\prime} \leq k$ ) and

$$
G^{k} h w \sqsubseteq G^{k-k^{\prime}} h w
$$

Now let

$$
k^{\prime}=\varrho_{m}\left(g, h\left(g_{1}^{k}(w)\right)\right) \cdot \varrho_{m}\left(g_{1}, w\right)
$$

As in the proof of Lemma 22 we have

$$
\begin{aligned}
& k-k^{\prime} \geq \ell_{m}\left(g_{1}, w\right)-\varrho_{m}\left(g_{1}, w\right) \\
& k-k^{\prime} \geq \ell_{m}\left(g, h\left(g_{1}^{k}(w)\right)\right)-\varrho_{m}\left(g, h\left(g_{1}^{k}(w)\right)\right)
\end{aligned}
$$

Using Fact 27 and the monotonicity of $g$ and $h$ we get

$$
\begin{aligned}
G^{k} h w & =g^{k}\left(h\left(g_{1}^{k}(w)\right)\right) \\
& =g^{k-k^{\prime}}\left(h\left(g_{1}^{k}(w)\right)\right) \\
& =g^{k-k^{\prime}}\left(h\left(g_{1}^{k-k^{\prime}}(w)\right)\right) \\
& =G^{k-k^{\prime}} h w
\end{aligned}
$$

This completes the proof.

It is now easy to see that Lemma 22 can be strengthened to show that if $G:\left(A \rightarrow_{t} B\right) \rightarrow\left(A \rightarrow_{t} B\right)$ is defined by $G h=g \circ h \circ g_{1}$ for $g \in A \rightarrow_{m} B$ then $G$ is $\ell_{m}(g) \cdot \ell_{t}\left(g_{1}\right)$-bounded. Knowledge of $g_{1} \in A \rightarrow_{m} B$ cannot be used because the argument to $G$ need not be monotone.

Example 28: Consider once again the detection of signs analysis of Example 21. The function

$$
\begin{gathered}
f x=\text { if } \operatorname{sq} x>100 \text { then } x \\
\text { else } \operatorname{sq}(f(x-3))
\end{gathered}
$$


gives rise to a functional

$$
H:(\mathbf{S} \rightarrow \mathbf{S}) \rightarrow(\mathbf{S} \rightarrow \mathbf{S})
$$

in linear form, i.e.

$$
H h=g_{0} \sqcup g \circ h \circ g_{1}
$$

The functions $g_{0}, g$ and $g_{1}$ may be defined by

\begin{tabular}{|l||cccccccc|}
\hline$x$ & $\perp$ & + & 0 & - & +0 & +- & $0-$ & $\top$ \\
\hline \hline$g_{0}(x)$ & $\perp$ & + & $\perp$ & - & +0 & +- & $0-$ & $\top$ \\
$g(x)$ & $\perp$ & + & 0 & + & +0 & + & +0 & $\top$ \\
$g_{1}(x)$ & $\perp$ & $\top$ & - & - & $\top$ & $\top$ & - & $\top$ \\
\hline
\end{tabular}

Note that $g$ is strict and additive so that Lemma 11 applies. We get

$$
\begin{aligned}
& \ell_{t}(g)=\ell_{m}(g)=2 \\
& \ell_{t}\left(g_{1}\right)=\ell_{m}\left(g_{1}\right)=2
\end{aligned}
$$

so that both Lemmas 22 and 25 yield a bound of 4 on the number of iterations needed. This is substantially better than the results of [9] where we obtain a bound of 9 (when using that the detection of signs analysis is in the strict and additive framework).

The third case is where $\varphi$ is $s a$. Here we would like to show that $G$ is $\ell_{s a}(g) \cdot \ell_{s a}\left(g_{1}\right)$-bounded but so far we have been unable to do so. Also we would like to strengthen Lemmas 22 and 25 to $\ell_{s a}(g) \cdot \ell_{t}\left(g_{1}\right)$ and $\ell_{s a}(g) \cdot \ell_{m}\left(g_{1}\right)$, respectively, provided that $g \in B \rightarrow_{s a} B$.

\section{Primitive recursive forms}

We now study a functional $G:\left(A \rightarrow_{\varphi} B\right) \rightarrow\left(A \rightarrow_{\varphi} B\right)$ defined by

$$
G h=g \circ \operatorname{tuple}\left(h \circ g_{1}, g_{2}\right)
$$


where $g \in B \times B \rightarrow_{\varphi} B, g_{1} \in A \rightarrow_{\varphi} A$ and $g_{2} \in A \rightarrow_{\varphi} B$. As already noted in Section 3, $G$ is strict and additive if $g$ is strict and additive in its left argument.

Rather than embarking on a detailed study of the iterands of

$$
H=\lambda h . g_{0} \sqcup(G h)
$$

we shall transform $H$ into another functional $H^{\prime}$, by using the well-known program transformation technique of "introducing an accumulator" [2, Section 6]. The functional $H^{\prime}:\left(A \times B \rightarrow_{\varphi} B\right) \rightarrow\left(A \times B \rightarrow_{\varphi} B\right)$ will be in iterative form and is defined by

$$
\begin{aligned}
& H^{\prime} h^{\prime}=g_{0}^{\prime} \sqcup\left(G^{\prime} h^{\prime}\right) \\
& G^{\prime} h^{\prime}=h^{\prime} \circ g_{1}^{\prime}
\end{aligned}
$$

where $g_{0}^{\prime} \in A \times B \rightarrow_{\varphi} B$ and $g_{1}^{\prime} \in A \times B \rightarrow_{\varphi} A \times B$ are defined by

$$
\begin{aligned}
& g_{0}^{\prime}=g \circ \operatorname{tuple}\left(g_{0} \circ f s t, \text { snd }\right) \\
& g_{1}^{\prime}=\operatorname{tuple}\left(g_{1} \circ f s t, g \circ \operatorname{tuple}\left(g_{2} \circ f s t, s n d\right)\right)
\end{aligned}
$$

The formal relationship between $H$ and $H^{\prime}$ is expressed by

Fact 29: Assume that $g \in B \times B \rightarrow_{\varphi} B$ satisfies

- $g$ is associative, i.e. $g\left(w_{1}, g\left(w_{2}, w_{3}\right)\right)=g\left(g\left(w_{1}, w_{2}\right), w_{3}\right)$, and

- $g$ is strict and additive in its left argument.

Then

$$
H^{\prime i} \perp\left(w, w_{1}\right)=g\left(H^{i} \perp w, w_{1}\right)
$$

holds for all $i \geq 0, w \in A$ and $w_{1} \in B$.

Proof: We prove the equality by numerical induction on $i$. The base case, when $i=0$, is immediate as $g$ is strict in its left argument.

For the induction step we calculate 


$$
\begin{aligned}
H^{\prime i+1} \perp\left(w, w_{1}\right) & =g_{0}^{\prime}\left(w, w_{1}\right) \sqcup H^{i} \perp\left(g_{1}^{\prime}\left(w, w_{1}\right)\right) \\
& =g\left(g_{0}(w), w_{1}\right) \sqcup H^{\prime i} \perp\left(g_{1}(w), g\left(g_{2}(w), w_{1}\right)\right) \\
& =g\left(g_{0}(w), w_{1}\right) \sqcup g\left(H^{i} \perp\left(g_{1}(w)\right), g\left(g_{2}(w), w_{1}\right)\right) \\
& =g\left(g_{0}(w), w_{1}\right) \sqcup g\left(g\left(H^{i} \perp\left(g_{1}(w)\right), g_{2}(w)\right), w_{1}\right) \\
& =g\left(g_{0}(w) \sqcup g\left(H^{i} \perp\left(g_{1}(w)\right), g_{2}(w)\right), w_{1}\right) \\
& =g\left(H^{i+1} \perp w, w_{1}\right)
\end{aligned}
$$

This completes the proof of the equality.

Fact 30: Assume that $g \in B \times B \rightarrow_{\varphi} B$ is associative, strict and additive in its left argument and that

- $g$ has a right-identity $w_{0} \in B$, i.e. $g\left(w_{1}, w_{0}\right)=w_{1}$.

We then have

- $H^{i} \perp w=H^{i} \perp\left(w, w_{0}\right)$

- $F I X H w=F I X H^{\prime}\left(w, w_{0}\right)$

for all $i \geq 0$ and $w \in A$.

Proof: Using that $w_{0}$ is a right identity we get from Fact 29 that

$$
H^{\prime i} \perp\left(w, w_{0}\right)=g\left(H^{i} \perp w, w_{1}\right)=H^{i} \perp w
$$

It then follows that $F I X H^{\prime}\left(w, w_{0}\right)=F I X H w$.

Fact 30 relates the fixed points of the two functionals but we shall also be interested in determining the number of unfoldings needed to compute the fixed points. We have

Lemma 31: Assume that $g$ is associative, has a right identity and is strict and additive in its left argument. If $k$ unfoldings suffice for $H^{\prime}$ then also $k$ unfoldings suffice for $H$, i.e.

$$
\text { FIX } H^{\prime}=H^{\prime k} \perp \text { implies FIX } H=H^{k} \perp
$$


In particular, FIX $H=H^{k} \perp$ if $G^{\prime}$ is $k$-bounded.

Proof: Assume that

$$
F I X \quad H^{\prime}=H^{\prime k} \perp
$$

For arbitrary $w \in A$ we then calculate

$$
\begin{aligned}
\text { FIX } H w & \left.=F I X H^{\prime}\left(w, w_{0}\right)\right) \\
& =H^{\prime k} \perp\left(w, w_{0}\right) \\
& =H^{k} \perp w
\end{aligned}
$$

where $w_{0}$ is the right identity of $g$ and we have used Fact 30 twice. For the final claim we simply use Lemma 11.

A similar result holds for the bounds of the functionals $G$ and $G^{\prime}$.

Lemma 32: Assume that $g$ is associative, has a right identity and is strict and additive in its left argument. Then $G$ and $G^{\prime}$ are strict and additive and

$G^{\prime}$ is $k$-bounded implies $G$ is $k$-bounded.

Proof Sketch: Note that we never made any assumptions about $g_{0}$, except $g_{0} \in A \rightarrow_{\varphi} B$, and that the definition of $G$ and $G^{\prime}$ is independent of $g_{0}$. Write

$$
\begin{aligned}
H\left[h_{0}\right] & =\lambda h . h_{0} \sqcup(G h) \\
H^{\prime}\left[h_{0}\right] & =\lambda h^{\prime} .\left(g \circ \operatorname{tuple}\left(h_{0} \circ f s t, s n d\right)\right) \sqcup\left(G^{\prime} h^{\prime}\right)
\end{aligned}
$$

for arbitrary $h_{0} \in A \rightarrow_{\varphi} B$. Next assume that $G^{\prime}$ is $k$-bounded.

We then have

$$
\begin{aligned}
\left(H^{\prime}\left[h_{0}\right]\right)^{k+1}(\perp) & =G^{[k+1]}\left(h_{0}\right) \\
& =G^{\prime[k]}\left(h_{0}\right) \\
& =\left(H^{\prime}\left[h_{0}\right]\right)^{k}(\perp) \\
& =\operatorname{FIX}\left(H^{\prime}\left[h_{0}\right]\right)
\end{aligned}
$$

using an analogue of Fact 10 and Lemma 11. Using an analogue of Lemma 31 we have 


$$
\left(H\left[h_{0}\right]\right)^{k+1}(\perp)=\left(H\left[h_{0}\right]\right)^{k}(\perp)=F I X\left(H\left[h_{0}\right]\right)
$$

and by analogues of Fact 10 we have

$$
\begin{aligned}
\left(H\left[h_{0}\right]\right)^{k+1}(\perp) & =G^{[k+1]}\left(h_{0}\right) \\
\left(H\left[h_{0}\right]\right)^{k}(\perp) & =G^{[k]}\left(h_{0}\right)
\end{aligned}
$$

It then follows that

$$
G^{[k+1]}\left(h_{0}\right)=G^{[k]}\left(h_{0}\right)
$$

for all $h_{0} \in A \rightarrow_{\varphi} B$ and this shows that $G$ is $k$-bounded.

Example 33: Consider the factorial function defined by

$$
\text { fac } \mathrm{n}=\text { if } \mathrm{n}=0 \text { then } 1 \text { else } \mathrm{n} * \operatorname{fac}(\mathrm{n}-1)
$$

A strictness analysis (along the lines of the Appendix) will give rise to a functional

$$
H:\left(2 \rightarrow_{m} 2\right) \rightarrow\left(2 \rightarrow_{m} 2\right)
$$

in primitive recursive form and with

$$
\begin{aligned}
& g_{0}(n \#)=1 \\
& g_{1}=g_{2}=i d \\
& g\left(n^{\#}, m^{\#}\right)=n^{\#} \sqcap m^{\#}
\end{aligned}
$$

To bound the number of iterations needed to compute the fixed point of $H$ it follows from Lemmas 31 and 16 that we only have to determine $\ell_{m}\left(g_{1}^{\prime}\right)$. We have

$$
\begin{aligned}
g_{1}^{\prime}\left(n^{\#}, m^{\#}\right) & =\operatorname{tuple}\left(g_{1} \circ f s t, g \circ \operatorname{tuple}\left(g_{2} \circ f s t, s n d\right)\right)\left(n^{\#}, m^{\#}\right) \\
& =\left(n^{\#}, n^{\#} \sqcap m^{\#}\right)
\end{aligned}
$$


Since $g_{1}^{\prime}$ is reductive we have $\ell_{m}\left(g_{1}^{\prime}\right)=1$.

This is clearly the optimal result. It is worth observing that it is also better than the bound of 2 obtainable from [9, Section 2]. Finally we should point out that the functional $H^{\prime}$ of the present example corresponds rather closely to the functional $H$ of Examples 17 and 14. (Exact correspondance fails because $g_{0}$ is not strict.)

\section{Conclusion}

We have considered the problem of bounding the number of iterations needed to compute the fixed point of a continuous functional

$$
H:(A \rightarrow B) \rightarrow(A \rightarrow B)
$$

defined on finite complete lattices $A$ and $B$. We have considered three defining forms of $H$ :

- iterative forms: $H h=g_{0} \sqcup\left(h \circ g_{1}\right)$

- linear forms: $H h=g_{0} \sqcup\left(g \circ h \circ g_{1}\right)$

- primitive recursive forms: $H h=g_{0} \sqcup\left(g \circ \operatorname{tuple}\left(h \circ g_{1}, g_{2}\right)\right)$

and three classes of functions from $A$ to $B$ :

- total functions

- monotone functions

- strict and additive functions

A related study was conducted in [9]. However, the main difference is that the bounds of [9] depended on measures of $A$ and $B$ whereas the bounds established here depend on measures of the functions $g_{1}$ etc. The results of the present paper may therefore carry over to the situation where neither $A$ nor $B$ are finite complete lattices. 
The following table summarizes all the information that can be obtained by combining the results established here with those of [9]. In the formulae using min we have arranged it so that the first argument is the result of the present paper and the second argument is the result from [9].

\begin{tabular}{|l||c|c|c|}
\hline & $A \rightarrow_{t} B$ & $A \rightarrow_{m} B$ & $A \rightarrow_{s a} B$ \\
\hline \hline iterative form & $\ell_{t}\left(g_{1}\right) \leq \mathbf{C}(A)$ & $\ell_{m}\left(g_{1}\right) \leq \mathbf{C}(A)$ & $\ell_{s a}\left(g_{1}\right) \leq \mathbf{C}(A)$ \\
\hline linear form & $\min \left\{\ell_{t}(g) \cdot \ell_{t}\left(g_{1}\right)\right.$, & $\min \left\{\ell_{m}(g) \cdot \ell_{m}\left(g_{1}\right)\right.$, & $\mathbf{R J C}(A) \cdot \mathbf{H}(B)$ \\
$(g$ restricted $)$ & $\mathbf{C}(A) \cdot \mathbf{H}(B)\}$ & $\mathbf{C}(A) \cdot \mathbf{H}(B)\}$ & \\
\hline prim. rec. form & $\operatorname{Imin}\left\{\ell_{t}\left(g_{1}^{\prime}\right)\right.$, & $\min \left\{\ell_{m}\left(g_{1}^{\prime}\right)\right.$, & $\min \left\{\ell_{s a}\left(g_{1}^{\prime}\right)\right.$, \\
$(g$ restricted $)$ & $\mathbf{C}(A) \cdot \mathbf{H}(B)\}$ & $\mathbf{C}(A) \cdot \mathbf{H}(B)\}$ & $\mathbf{R J C}(A) \cdot \mathbf{H}(B)$, \\
& & & $\mathbf{H}(A)+\mathbf{H}(B)\}$ \\
\hline no restriction & $\mathbf{C}(A) \cdot \mathbf{H}(B)$ & $\mathbf{C}(A) \cdot \mathbf{H}(B)\}$ & $\mathbf{R J C}(A) \cdot \mathbf{H}(B)$ \\
\hline
\end{tabular}

We should explain that RJC $(A)$ is the number of non-bottom join-irreducible elements of $A$; if $A$ is distributive we have $\mathbf{R J C}(A)=\mathbf{H}(A)$ and in general $\mathbf{H}(A) \leq \mathbf{R J C}(A) \leq \mathbf{C}(A)$. Finally we should remark that the $\mathbf{H}(A)+\mathbf{H}(B)$ entry in the table follows from $\ell_{s a}\left(g_{1}^{\prime}\right) \leq \mathbf{H}(A \times B)$ where we use the results already established for iterative forms.

Thus for the iterative forms the bounds of the present paper will always be at least as good as those of [9] whereas this need not be the case for linear forms and primitive recursive forms. However, the main point is that in the "average" case we expect $\ell_{t}\left(g_{1}\right)$ to be muchless than $\mathbf{C}(A)$ etc., so that in the "average" case we are likely always to get an improvement over [9]. This suggests studying certain analyses, e.g. corresponding to fast analyses, where this always can be guaranteed.

\section{Acknowledgements}

The insights needed to write this paper were greatly assisted by Henrik Andersen's talk on model checking at the First DART-Workshop [7] and by subsequent discussions with him. This research has been supported in part by The Danish Research Councils, under the DART-project. 


\section{References}

[1] A. V. Aho, R. Sethi, J.D. Ullman: Compilers - Principles, Techniques and Tools, Addison - Wesley (1986).

[2] R.M. Burstall, J. Darlington: A Transformation System for Developing Recursive Programs, Journal of the ACM 241 (1977).

[3] A.J. Field, P.G. Harrison: Functional Programming, Addison - Wesley (1988).

[4] G. Grätzer: Lattice Theory - First Concepts and Distributive Lattices, W.H. Freeman and Company (1971).

[5] T.J. Marlowe, B.G. Ryder: Properties of Data Flow Frameworks - A Unified Model, Acta Informatica 28 (1990).

[6] A. Mycroft: Abstract Interpretation and Optimizing Transformations for Applicative Programs, University of Edinburgh Ph.D.-thesis (1981).

[7] F. Nielson (editor): Design, Analysis and Reasoning about Tools: Abstracts from the First Workshop, Aarhus University report DAIMI PB367 (1991).

[8] F. Nielson: Two-Level Semantics and Abstract Interpretation, Theoretical Computer Science 69 (1989).

[9] H.R. Nielson, F. Nielson: Bounded Fixed Point Iteration, Proceedings of the ACM Symposium on Principles of Programming Languages (1992).

[10] F. Nielson, H.R. Nielson: Finiteness Conditions for Fixed Point Iteration (Extended Abstract), Proceedings of the ACM Sympoosium on LISP and finctional Programming (to appear 1992).

[11] H.R. Nielson, F. Nielson: Transformations on Higher-Order Functions, Proceedings FPCA'89 (1989).

[12] P. Wadler, R.J.M. Hughes: Projections for Strictness Analysis, Proceedings FPCA'87, Springer Lecture Notes in Computes science 274 (1987). 


\section{Appendix}

In this Appendix we shall claim that the results of the present paper are widely applicable because the special forms allowed for the functionals $H$ and $G$ are likely to arise frequently. The general idea is that the functional $H$ will be

- in iterative form if the function being analysed is tail recursive (corresponding to an iterative loop),

- in linear form if the function being analysed contains only one recursive call in its defining equation (somewhat analogous to the linear forms of $[3])$,

- in primitive recursive form if the function being analysed is primitive recursive.

To be able to substantiate these claims we must make several assumptions on how we analyse the various primitives. To this end we shall assume that

- function composition is interpreted as function composition (so that only forward analyses are considered),

- tupling is interpreted as tupling meaning that the abstraction of a pair is a pair of abstractions ${ }^{3}$,

- the conditional given by

$$
\operatorname{cond}\left(p, f_{1}, f_{2}\right) v= \begin{cases}f_{1}(v) & \text { if } p(v)=\text { true } \\ f_{2}(v) & \text { if } p(v)=\text { false } \\ \perp & \text { otherwise }\end{cases}
$$

is interpreted as

$$
\text { cond }^{\#}\left(p^{\#}, f_{1}^{\#}, f_{2}^{\#}\right)=\left(f_{1}^{\#} \circ p_{\text {true }}^{\#}\right) \sqcup\left(f_{2}^{\#} \circ p_{\text {false }}^{\#}\right)
$$

\footnotetext{
3 "No tensor products."
} 
where $p_{\text {true }}^{\#}$ and $p_{\text {false }}^{\#}$ are filters defined from $p^{\#}$ so that typically

$$
p_{b}^{\#} w= \begin{cases}w & \text { if } p^{\#}(w) \text { is an abstraction of } b \\ \perp & \text { otherwise }\end{cases}
$$

Under these assumptions we can validate our claims as illustrated below.

Example A1: A tail recursive function has the general form

$$
\begin{aligned}
f x & =\text { if } p x \text { then } f_{1} x \text { else } f\left(f_{2} x\right) \\
& =\operatorname{cond}\left(p, f_{1}, f \circ f_{2}\right) x
\end{aligned}
$$

The functional $H$ obtained from the analysis will then be

$$
\begin{aligned}
H h & =\operatorname{cond}^{\#}\left(p^{\#}, f_{1}^{\#}, h \circ f_{2}^{\#}\right) \\
& =\left(f_{1}^{\#} \circ p_{\text {true }}^{\#}\right) \sqcup h \circ\left(f_{2}^{\#} \circ p_{\text {false }}^{\#}\right)
\end{aligned}
$$

which is in iterative form so that the results of Section 4 apply.

Example A2: For us a linear function has the general form

$$
\begin{aligned}
f x & =\text { if } p x \text { then } f_{1} x \text { else } f_{2}\left(f\left(f_{3} x\right)\right) \\
& =\operatorname{cond}\left(p, f_{1}, f_{2} \circ f \circ f_{3}\right) x
\end{aligned}
$$

The functional $H$ obtained from the analysis is then

$$
\begin{aligned}
H h & =\operatorname{cond}^{\#}\left(p^{\#}, f_{1}^{\#}, f_{2}^{\#} \circ h \circ f_{3}^{\#}\right) \\
& =\left(f_{1}^{\#} \circ p_{\text {true }}^{\#}\right) \sqcup\left(f_{2}^{\#} \circ h \circ\left(f_{3}^{\#} \circ p_{\text {false }}^{\#}\right)\right)
\end{aligned}
$$

which is in linear form so that the results of Section 5 apply.

Example A3: A primitive recursive function has the general form

$$
\begin{aligned}
f x & =\text { if } p x \text { then } f_{1} x \text { else } f_{2}\left(f\left(f_{3} x\right), x\right) \\
& =\operatorname{cond}\left(p, f_{1}, f_{2} \circ \operatorname{tuple}\left(f \circ f_{3}, i d\right)\right) x
\end{aligned}
$$

The functional $H$ obtained from the analysis is then 


$$
\begin{aligned}
H h & =\operatorname{cond}^{\#}\left(p^{\#}, f_{1}^{\#}, f_{2}^{\#} \circ \operatorname{tuple}\left(h \circ f_{3}^{\#}, i d^{\#}\right)\right) \\
& =\left(f_{1}^{\#} \circ p_{\text {true }}^{\#}\right) \sqcup\left(f_{2}^{\#} \circ \operatorname{tuple}\left(h \circ\left(f_{3}^{\#} \circ p_{\text {false }}^{\#}\right), i d^{\#} \circ p_{\text {false }}^{\#}\right)\right)
\end{aligned}
$$

which is in primitive recursive form so that the results of Section 6 do apply.

The more debatable restriction on the form of the analyses is probably that for the conditional. One may note that it holds in classical flow analysis [1] as well as in many instances of abstract interpretation [8]. For simple strictness analysis [6] over the two point domain it is more common to have

$$
\operatorname{cond}^{\prime}\left(h, h_{1}, h_{2}\right) w=h(w) \sqcap\left(h_{1}(w) \sqcup h_{2}(w)\right)
$$

Since the lattices of concern are distributive we have

$$
\operatorname{cond}^{\prime}\left(h, h_{1}, h_{2}\right) w=\left(h(w) \sqcap h_{1}(w)\right) \sqcup\left(h(w) \sqcap h_{2}(w)\right)
$$

If $h_{1}$ and $h_{2}$ are strict we can bring cond' into the desired form by setting

$$
\operatorname{cond}^{\prime}\left(h, h_{1}, h_{2}\right) w=h_{1}\left(h_{\text {true }}(w)\right) \sqcap h_{2}\left(h_{\text {false }}(w)\right)
$$

where

$$
h_{\text {true }}(w)=h_{\text {false }}(w)= \begin{cases}w & \text { if } h(w)=1 \\ \perp & \text { otherwise }\end{cases}
$$

When $h_{1}$ and $h_{2}$ are not strict one may change the analysis to include a new "artificial" $\perp$-element in which all functions are strict. This is similar to the approach of projection based strictness analysis [12].

Finally we should like to stress that the requirements on the analyses and functions are sufficient but not necessary in order to apply the results.

Example A4: Consider strictness analysis of the Fibonacci function

$$
\text { fib } n=\text { if } n \leq 1 \text { then } 1 \text { else fib }(n-1)+f i b(n-2)
$$


Even though this function is not in "linear form" the corresponding functional may be simplified to one that is in iterative form.

Example A5: Also the restrictions on the analyses can sometimes be lifted. In a backward analysis it is often natural to take

$$
\begin{aligned}
h_{1} \circ^{\#} h_{2} & =h_{2} \circ h_{1} \\
\operatorname{cond}^{\#}\left(h, h_{1}, h_{2}\right) w & =h_{1}(w) \sqcup h_{2}(w) \sqcup h(1) \\
\operatorname{tuple}^{\#}\left(h_{1}, h_{2}\right)\left(w_{1}, w_{2}\right) & =h_{1}\left(w_{1}\right) \sqcup h_{2}\left(w_{2}\right)
\end{aligned}
$$

If we apply this analysis to a primitive recursive function we will obtain a functional in linear form. An example of an analysis satisfying these conditions is the liveness analysis of [11]. 